\title{
La microscopie à force atomique dans la recherche en biologie
}

Le microscope à force atomique est un nouveau type d'instrument permettant une résolution à l'échelle atomique de cristaux et, à l'échelle moléculaire, de certaines substances biologiques. Le principe en est le balayage de la surface des objets étudiés à l'aide d'une micropointe dont les mouvements sont analysés par ordinateur. Cette opération peut se dérouler en milieu liquide, ce qui augmente l'intérêt de cet appareil pour étudier des structures ou des processus biologiques. Quoique les artefacts possibles soient encore nombreux, les développements techniques rapides dont bénéficie cet instrument en font un outil des plus prometteurs.

\section{Sandor Kasas}

\section{ADRESSE}

S. Kasas : docteur en médecine. Institut d'histologic ct d'cmbryologie générale (directeur : pr. M.R. Cclio), route A. Gackel, CH1700 e microscope à force atomique (AFM) est un nouveau type de microscope permettant d'atteindre une résolution atomique sur des échantillons inorganiques et une résolution moléculaire sur des spécimens biologiques. Les groupes de recherche qui l'utilisent en biologie sont de plus en plus nombreux et les images produites par AFM inondent la littérature scientifique. Utilisant ce microscope depuis bientôt une année, nous avons voulu, dans cet article, non seulement souligner les possibilités actuelles et les promesses de cet instrument, mais également montrer ses limites et présenter quelques-uns de ses défauts.
En 1986, Gerd Binning et Heinrich Rohrer ont reçu le prix Nobel de physique pour l'invention du microscope à eff et tunnel (STM). Ils avaient démontré que ce microscope pouvait visualiser les atomes avec une résolution jusque-là inégalée [1]. Se fondant sur le principe du STM, de nombreux chercheurs mirent au point un certain nombre de microscopes similaires, aujourd'hui globalement appelés scanning probe microscopes. Leur principe de fonctionnement est fondé sur l'exploration de l'échantillon par une sonde de petite taille, sensible à la température, au champ magnétique, à la transparence, à la conductibilité ionique [2], ou à toute autre 
caractéristique physique ou électrique de l'échantillon. Le microscope dont la sonde exploratrice est sensible à la force d'interaction entre une pointe en diamant et l'échantillon, est appelé microscope à force atomique (AFM). Il fut inventé et mis au point par Binnig, Quate et Gerber en 1986 [3]. Il est actuellement, avec le STM, le scanning probe microscope le plus utilisé de par le monde.

\section{L'AFM et son principe de fonctionnement}

Le microscope [4] est de taille réduite: il ne dépasse pas $30 \mathrm{~cm}$ dans sa plus grande dimension. Étant très sensible aux vibrations extérieures, il est indispensable de le fixer sur un support muni d'amortisseurs : une table optique, par exemple.

Un ordinateur de type PC se charge de l'acquisition des images et du pilotage du microscope. Le principe de fonctionnement de l'AFM est simple : une pointe en diamant ou en silicium est fixée à l'extrémité d'un petit levier (figure 2, p. 142). Cette aiguille est abaissée jusqu'à ce qu'elle touche l'échantillon. Ce contact établi, l'ordinateur provoque le déplacement de l'échantillon de manière à effectuer un balayage de la surface du spécimen sous la pointe. Ce même ordinateur enregistre, par l'intermédiaire de capteurs divers, les déflexions verticales du levier. Un mécanisme de rétro-contrôle (en feedback) maintient la déflexion du levier constante en le déplaçant de bas en haut, suivant la topographie de l'échantillon. L'image de la surface du spécimen observé est obtenue en affichant tous les mouvements qui ont été imprimés à l'échantillon et au levier.

Un autre mode de fonctionnement consiste à maintenir le levier à hauteur constante et à enregistrer des déflexions de celui-ci lors des déplacements de l'échantillon. C'est ce mode de fonctionnement qui est illustré sur la figure 3 ( $p$. 143).

Si le principe de fonctionnement est globalement le même pour tous les AFM, les solutions techniques pour piloter le microscope et détecter les infimes déformations du levier varient de microscope en microscope. Les déplacements du levier (axe des $z$ ) et $m / s n^{\circ} 2$, vol. 8, féurier 92

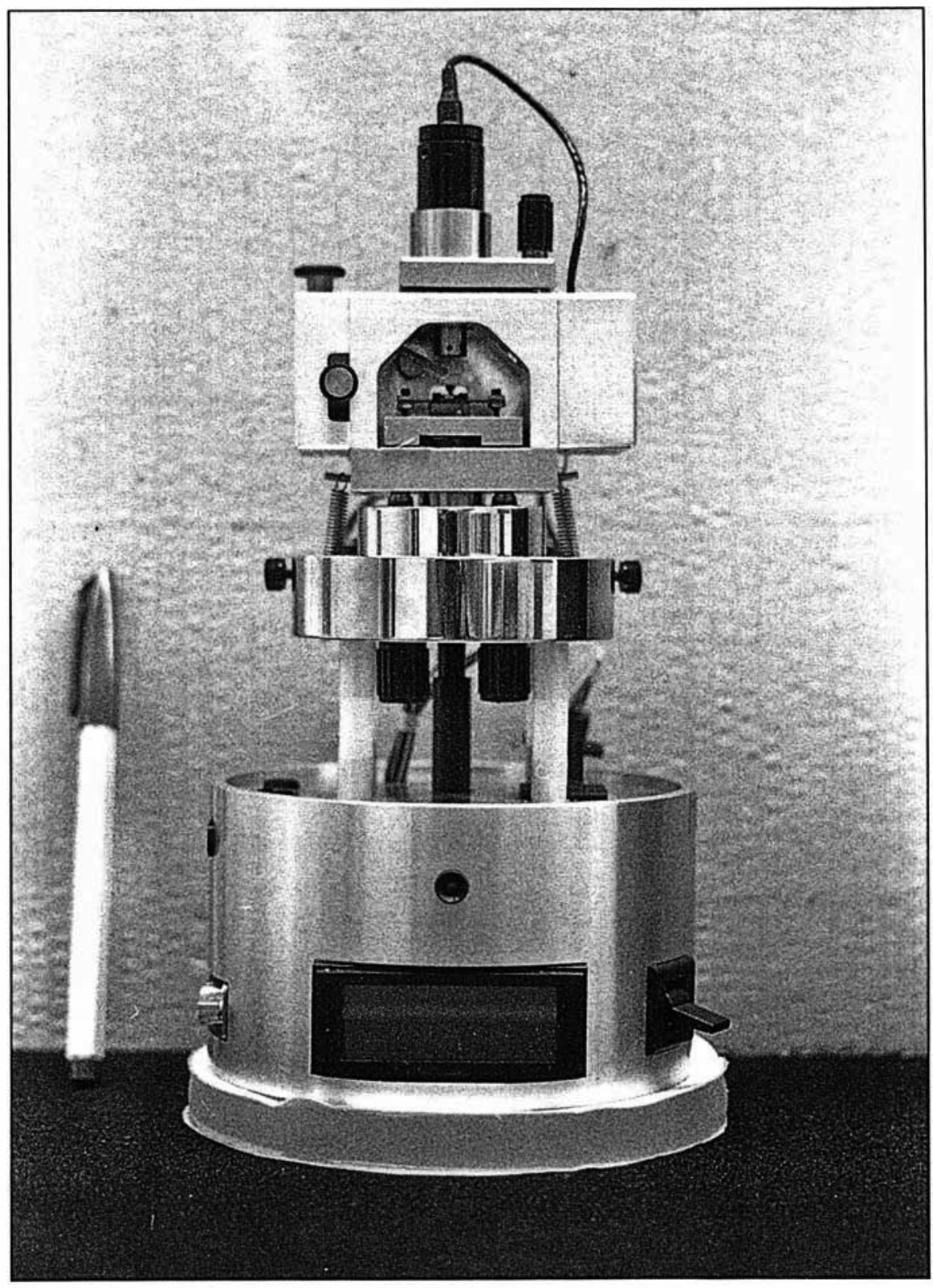

Figure 1. Le microscope à force atomique Nanoscope $\|^{\circledR}$.

de l'échantillon (axes des $x$ et des $y$ ) sont assurés par des cristaux piézoélectriques. Ceux-ci ont la particularité de se déformer lorsqu'on applique une tension à leur extrémité. En utilisant des cristaux piézo-électriques de petite taille, mus par des tensions faibles, on obtient des déplacements de l'ordre du centième de nanomètre permettant la résolution atomique (figure 4, p. 144).

Des cristaux plus longs, entraînés par des tensions plus élevées, permettent d'atteindre des champs de balayage de 80 à $120 \mu \mathrm{m}$ de diamètre.

L'AFM a la possibilité, très convoitée par les biologistes, de travailler dans les liquides [5-7]. Cela est rendu possible en enfermant le levier et l'échantillon dans une chambre hermétique à l'aide d'un joint torique. Il suffit par la suite de remplir cette chambre avec le liquide de son choix. Ce dispositif est illustré sur la figure 5 (p. 144).

Deux trous, sur les parties latérales 


\section{RÉFÉRENCES}

1. Binnig $\mathrm{G}$, Rohrer $\mathrm{H}$, Gerber $\mathrm{C}$, Weibel E. Surfacc studies by scanning tunncling microscopy. Phys Rev Lett 1982 ; 49 : 57-61.

2. Hansma PK, Drakc B, Marti O, Gould SAC, Prater CB. The scanning ionconductance microscopc. Science $1989 ; 243$ 641-3

3. Binnig G, Quate CF, Gcrber C. Atomic forcc microscopc. Phys Rev Lett 1986 ; 12 930-3

4. Digital Instruments Inc. 6780 Cortona Drive, Santa Barbara, California 93117

5. Marti O, Drakc B, Hansma PK. Atomic force microscopy of liquid-covered surfaccs : atomic rcsolution images. Appl Phys Lett 1987 ; 51 : 484-6.

6. Gould SAC, Drake B, Prater CB, et al From atoms to integrated-circuit chips blood cclls and bacteria with the atomic force microscopc. J Vac Sci Technol 1990 ; 8 . 369-73.

7. Egger M, Ohnesorge F, Weisenhorn AL, et al. Wet lipid-protein membranes imaged at submolecular resolution by atomic forcc microscopy. J Struct Biol 1990; $103: 89-94$

8. Amrcin M, Durr R, Stasiak A, Gross H, Travaglini G. Scanning tunncling microscopy of uncoatcd recA-DNA complexes. Science $1989 ; 243$ : 1708-11.

9. Edstrom RD, Mcinke $\mathrm{MH}$, Yang $\mathrm{X}$ Yang R, Elings V, Evans DF. Dircet visualization of phosphorylasc-phosphorylase kinasc complexes by scanning tunncling and atomic force microscopy. Biophys J 1990 $58: 1437-48$

10. Mcycr E, Howald L, Overncy RM, et al. Molccular-resolution images of Langmuir-Blodgett films using atomic force microscopy. Nature 1991; 349 : 398-400.

11. Weiscnhorn AL, Gaub HE, Hansma HG, Sinsheimer RL, Kclderman GL, Hansma PK. Imaging single-stranded DNA, antigen-antibody reaction and polymerized Langmuir-Blodgett films with an atomic force microscope. Scann Microscopy $1990 ; 4$ $511-6$

12. Wciscnhorn AL, Drake B, Prater CB, et al. Immobilized protcins in buffer imaged at molccular resolution by atomic force microscopy. Biophys $J$ 1990; 58 : 1251-8.

13. Drakc B, Prater CB, Wcisenhorn AL, et al. Imaging crystals, polymers, and proccsses in water with the atomic forcc microscopc. Science $1989 ; 243: 1586-9$.

14. Kirk MD, Albrecht T, Quate CF. Lowtemperaturc atomic forcc microscopy. Rev $S c$

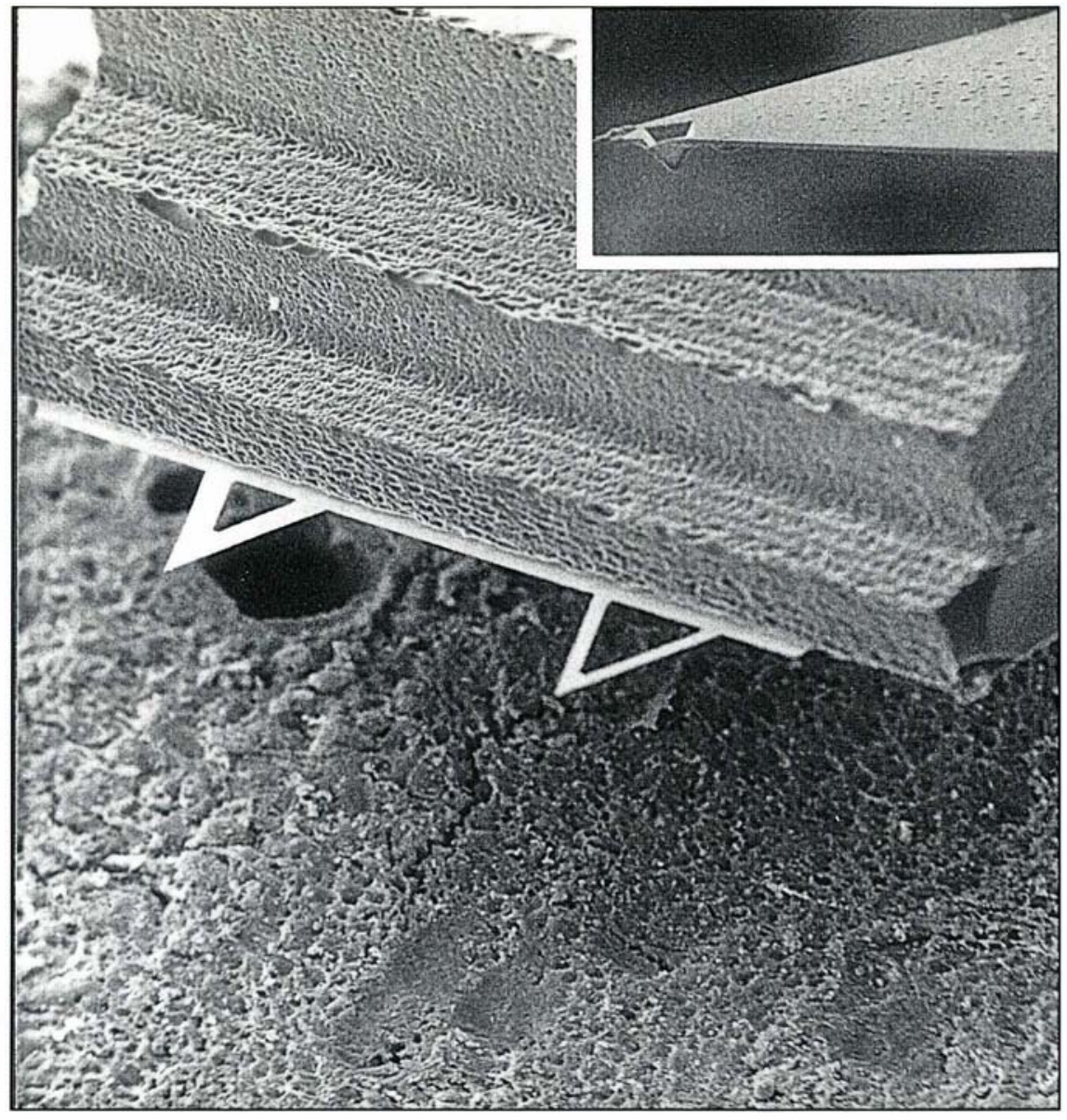

Figure 2. Image d'un levier d'AFM vu au microscope électronique à balayage $(30$ x). A l'extrémité du levier, et dirigée vers le bas, se trouve la pointe qui entre en contact avec l'échantillon (voir encadré, 270 x).

du dispositif suspenseur du levier, permettent de changer de liquide pendant l'acquisition de l'image.

\section{Avantages et désavantages de I'AFM vis-à-vis du STM}

Le principe de fonctionnement du STM diverge relativement peu de celui de l'AFM. Il s'agit également d'un scanning probe microscope, mais sa sonde exploratrice, une aiguille métallique terminée par un seul atome, analyse la conductibilité de l'échantillon, et non plus sa topographie. Une différence de potentiel est maintenue entre le spécimen à examiner et la sonde exploratrice. Lorsque l'aiguille est abaissée à quelques nanomètres au-dessus de l'échantillon, certains électrons traversent cette distance infinitésimale et engendrent un courant de quelques nanoampères dans l'aiguille. Ce phénomène est appelé " effet tunnel ", d'où le nom du microscope. Un mécanisme d'asservissement maintient le courant tunnel constant en variant la hauteur de l'aiguille lorsque celle-ci balaye la surface de l'échantillon. Comme dans l'AFM, toutes les déflexions de l'aiguille sont enregistrées dans un ordinateur et affichées à l'écran.

Pour pouvoir analyser l'image des spécimens biologiques avec le STM, il faut préalablement les rendre conducteurs, en vaporisant une couche de métal sur leur surface, par exemple. Cependant, si l'échantillon iso- 
lant est suffisamment mince et qu'il est posé sur un support conducteur, cette opération n'est plus nécessaire ; certains ćlectrons parviennent quand même jusqu'à l'aiguille et y engendrent un courant tunnel. C'est ainsi que des images d'échantillons non traités d'ADN ou de protéines ont pu être obtenues avec le STM [8].

Actuellement, la plupart des chercheurs travaillant avec des scanning probe microscopes préfèrent utiliser un AFM plutôt qu'un STM pour ćtablir des images d'objets biologiques, et cela pour plusicurs raisons : les images fournies par l'AFM ne dépendent que de la topographic de l'échantillon, alors que celles obtenues par le STM reflètent également sa conductibilité (figure 6, p. 145), l'observation directe d'échantillons isolants ćpais est impossible avec le STM, alors qu'elle ne présente aucun problème avec un AFM. Cependant, les images de protéines et d'ADN obtenues avec le STM semblent très prometteuses et suggèrent que le STM a certainement un brillant avenir dans le domaine de l'imagerie tridimensionnelle de molécules organiques.

\section{Les applications en biologie}

En tant que morphologistes, nous nous sommes intéressćs tout d'abord à l'utilisation de l'AFM pour visualiser des tissus préparés selon les méthodes classiques utilisées en microscopic. La figure 7 ( $p$. 145) montre une coupe semi-fine de muscle strié squelettique $(0,5 \mu \mathrm{m}$ d'épaisscur) obtenue à l'ultracryomicrotome, déposée sur une lame de verre et visualisée avec l'AFM à faible grossissement.

La technique de préparation utiliséc ne permet pas de dépasser la résolution du microscope électronique à transmission ; néanmoins, l'observation de ce type de préparation peut s'avérer utile pour comparer la même zone de l'échantillon vue avec l'AFM et le microscope électronique, le but de ces opérations étant de micux comprendre les images produites par l'AFM, souvent brouillées par des artefacts.

Pour un biologiste, la qualité la plus intéressante de l'AFM est sans doute sa possibilité de travailler en milieu liquide. La figure 8 (p. 145) montre $m / s n^{\circ} 2$, vol. 8, févier 92 un érythrocyte fixé au glutaraldéhyde et visualisé dans un tampon phosphate.

Bien que la mise en ocuvre de cette technique soit plus délicate que celle employéc pour la visualisation des échantillons dans l'air, nous pensons que cette méthode d'observation est la méthode de choix en biologic. Les forces d'interaction entre la pointe et le spécimen sont réduites au minimum (c'est-à-dire à des ordres de grandeur de $10^{-}{ }^{8}$ newton) et l'échantillon ne subit pratiquement pas de manipulations ; en plus, la "doulourcuse" phase de séchage, jusqu'ici inćvitable, lui est épargnée. La grande majorité des groupes de
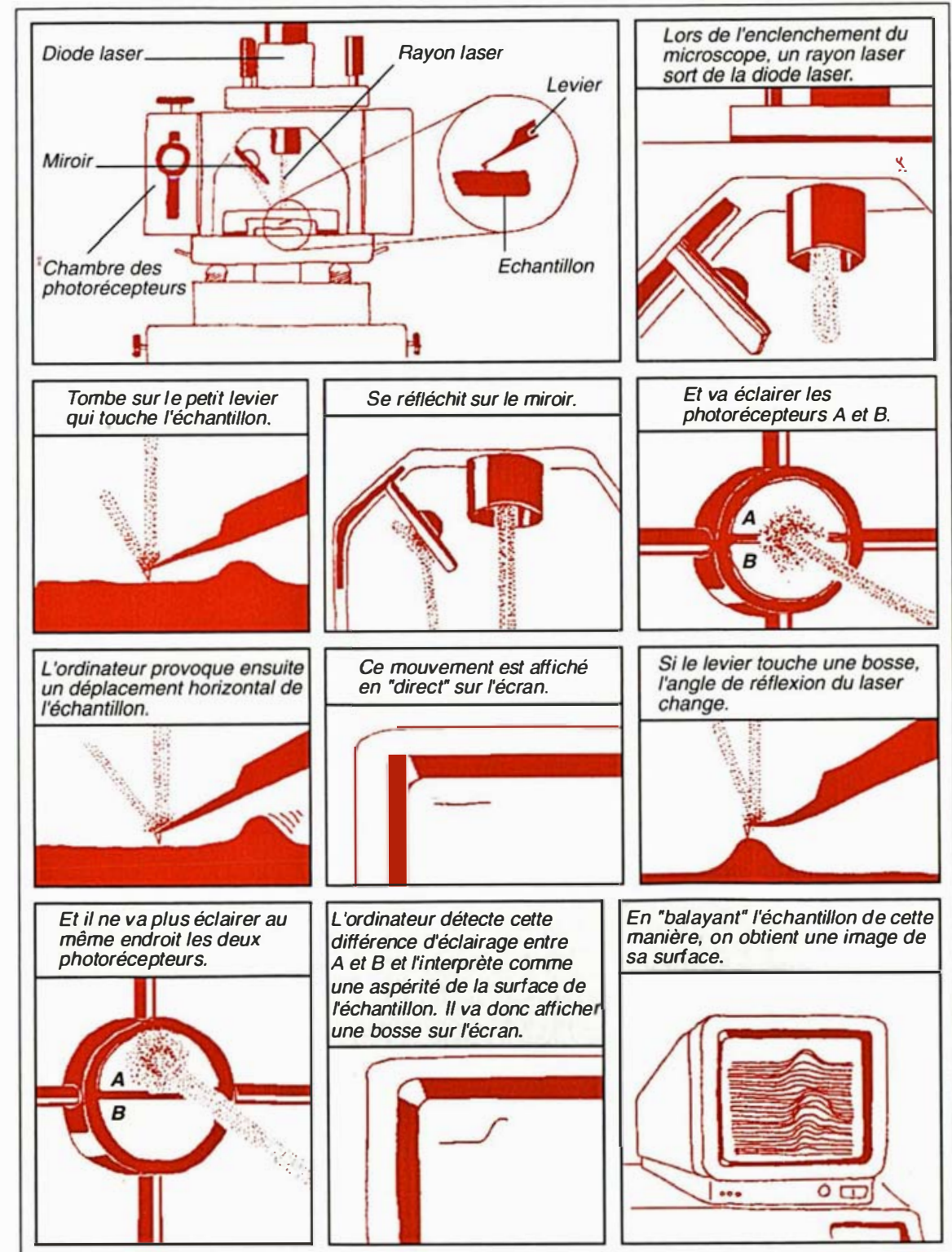
En "balayant" l'echantillon de cette
manière, on obtient une image de sa surface.

Figure 3. Principe de fonctionnement de I'AFM. Cette bande dessinée illustre le mode de fonctionnement appelé "hauteur constante ". Dans ce mode, le levier est maintenu à une hauteur fixe au-dessus de l'échantillon. Lors du balayage, les aspérités du spécimen déforment le levier et indiquent par ce biais la topographie de l'échantillon. L'autre mode de fonctionnement est appelé "force constante". Dans ce mode, lorsqu'une aspérité ou un trou déforment le levier, celui-ci est immédiatement relevé ou abaissé et ce sont les déplacements verticaux du levier qui renseignent l'ordinateur sur la topographie de l'échantillon. 


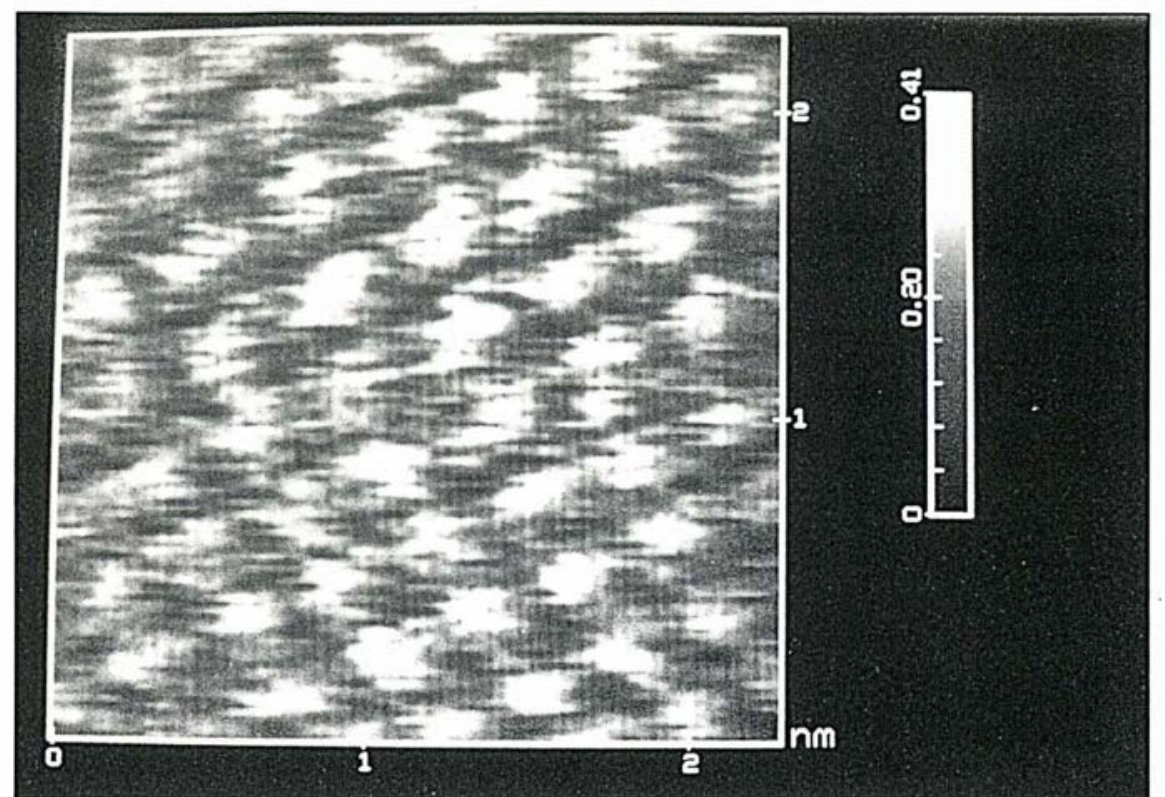

Figure 4. NaCl visualisé à résolution atomique. Les parties claires représentent les zones surélevées : ici elles correspondent aux atomes de Cl. Ceux de $\mathrm{Na}$, plus petits, sont invisibles sur cette image. Les graduations sur les bords droit et inférieur de l'image, ainsi que sur l'échelle, indiquent les dimensions en $\mathrm{nm}$.

recherche travaillant avec des AFM dans le domaine de la biologie essaie d'obtenir des images de molécules participant à la constitution de la matière vivante. Les images de protéines [9], de phospholipides [10] et d'ADN [11] qui ont été publićes se comptent par dizaines, souvent avec une résolution submoléculaire. Certains groupes synthćtisent des membranes de phospholipides, y incorporent des protéines et visualisent le tout avec l'AFM [12], le but de ces travaux étant d'observer une protéine incluse dans une membrane artificielle qui imite, aussi bien morphologiquement que physiologiquement, les membranes biologiques. Un certain nombre de groupes travaillent sur le séquençage de l'ADN et de l'ARN avec l'AFM dans l'espoir de rendre cette manipulation plus rapide et plus simple.
Figure 5 Dispositif permettant I'AFM de travailler dans un milieu liquide. 1 : liquide; 2 : échantillon 3 : joint torique; 4 : porte levier en Plexiglas transparent; 5 : rayon laser se réfléchissant sur le levier. Le dis positif comporte en outre deux ouvertures permettant à l'utilisateur de changer de liquide même lors du balayage.
Les figures 9 et 10 (p. 146) montrent une molécule de laminine visualisée dans notre laboratoire. La laminine est une protéine intervenant dans l'adhérence des cellules sur la membrane basale et la figure 11 (p. 147) illustre le modèle théorique de cette molécule.

Les figures 9 et 10 montrent la molécule de laminine visualisée avec une force de pression de levier de plus en plus grande. On voit clairement que la molécule est "pressée " contre le support de mica sur lequel elle repose. Ces images démontrent la possibilité non seulement de visualiser, mais aussi d'explorer les caractéristiques mécaniques d'une seule protéine. Il est ainsi possible de déterminer le coefficient d'élasticité de chaque nanomètre carré constituant la molécule.

Une autre voie de recherche est l'utilisation de l'AFM dans le suivi de processus dynamiques survenant à l'échelle moléculaire. Drake et al. [13] ont réussi à visualiser en direct la polymérisation de la fibrine avec une résolution moléculaire.

\section{Surfaces-support pour les échantillons biologiques}

Les surfaces-support utilisées pour adsorber les échantillons doivent remplir un certain nombre de conditions. La première est que la rugosité du support soit négligeable par rapport à la taille de l'échantillon. Si le spécimen est de taille importante (globule rouge, bactérie), une plaque de verre peut suffire, mais, en revanche, si l'on désire observer des molécules isolées, alors il devient indispensable d'utiliser un substrat qui soit atomiquement plat. Une deuxième condition est que la surface support soit hydrophile; il est ćgalement souhaitable qu'elle puisse être facilement nettoyée, aisément manipulable et peu onéreuse. Certains groupes utilisent du silicium amorphe, du graphite, du mica ou encore des grilles de microscopie électronique vaporisées avec du titane. Il est évident qu'il n'existe pas de support universel et que le choix se fait en fonction de l'échantillon. De nombreux groupes travaillent actuellement sur la recherche de procédés chimiques ou physiques qui permettraient de lier de manière covalente des molécules

$$
\mathrm{m} / \mathrm{s} n^{\circ} 2 \text {, vol. 8, février } 92
$$


organiques à un substrat atomiquement plat.

\section{Limites de I'AFM}

Il n'est pas d'instrument qui ne possède de défaut ou de limite, et l'AFM n'échappe pas à cette règle. Les imperfections que nous avons pu constater en utilisant cet appareil ne sont pas très nombreuses, mais il en est deux qui méritent d'être soulignćes. La première est que le champ de balayage maximal du microscope est limité à $80 \mu \mathrm{m}^{2}$. En biologic, où les préparations sont loin d'être homogènes, cette surface peut s'avérer parfois insuffisante pour une identification de la structure observée. L'autre défaut, tout aussi handicapant que le premier, est la limitation de la déflexion maximale du levier dans l'axe des $z$ à $5 \mu \mathrm{m}$. Cette faible valcur exclut l'observation d'échantillons un tant soit peu rugueux.

\section{- Les artefacts}

Si le principe de fonctionnement de l'AFM est simple, les artefacts qu'il engendre sont nombreux et leur explication souvent difficile. Ayant l'occasion d'en observer régulièrement, nous les avons classés de la manière suivante :

\section{- Artefacts dus à l'interaction entre la} pointe et le spécimen

Nous classons dans ce groupe toutes les structures qui sont créées, ou dont l'apparence est modifiée par l'interaction entre la pointe et l'échantillon. La figure 12 (p. 147) représente un

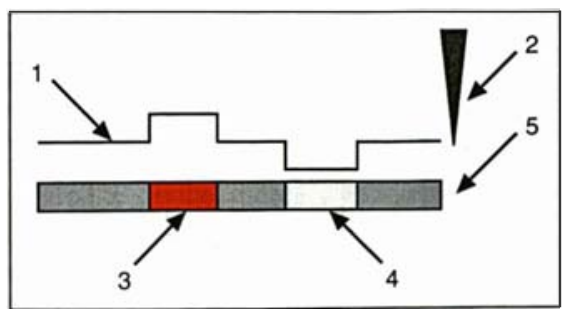

Figure 6. Les zones conductrices et isolantes de l'échantillon sont interprétées par les STM comme étant des aspérités de surface. 1 : trajectoire de l'aiguille ; 2 : aiguille du STM ; 3: zone fortement conductrice; 4 : zone faiblement conductrice; 5 : zone moyennement conductrice.

$m / s n^{\circ} 2$, vol. 8 , février 92

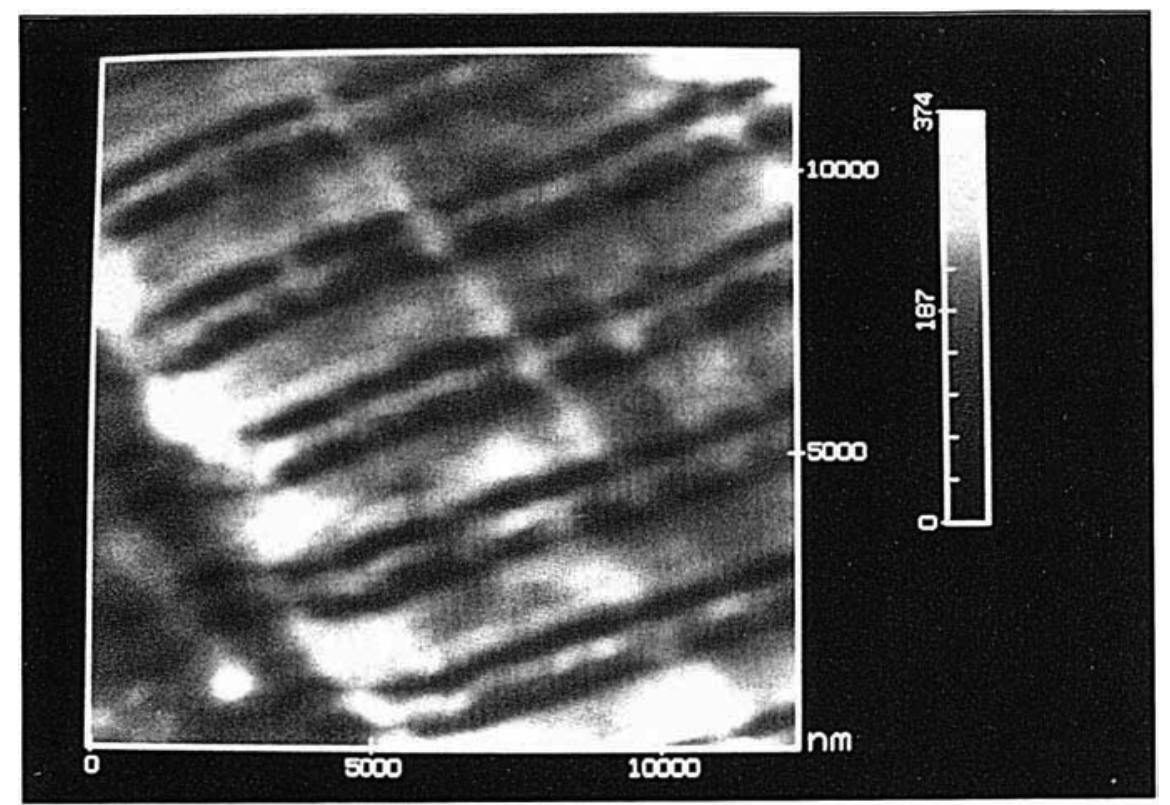

Figure 7. Musculature striée squelettique de rat coupée à I'ultracryomicrotome et visualisée dans I'air à l'aide de I'AFM. La striation épaisse correspond aux bandes $A$ et la striation mince aux lignes $Z$. Les graduations (sur l'image et l'échelle) indiquent les dimensions en $\mathrm{nm}$.

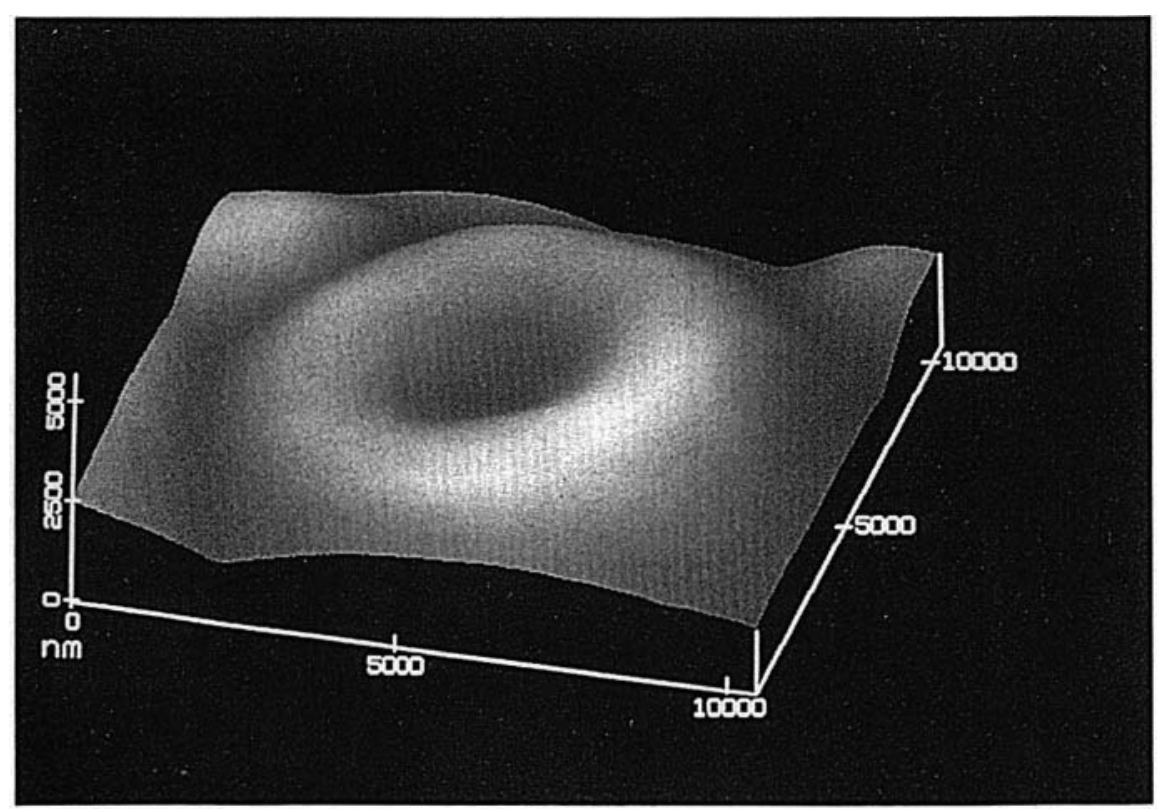

Figure 8. Érythrocyte visualisé dans un tampon physiologique. Graduations : dimensions en $\mathrm{nm}$. 


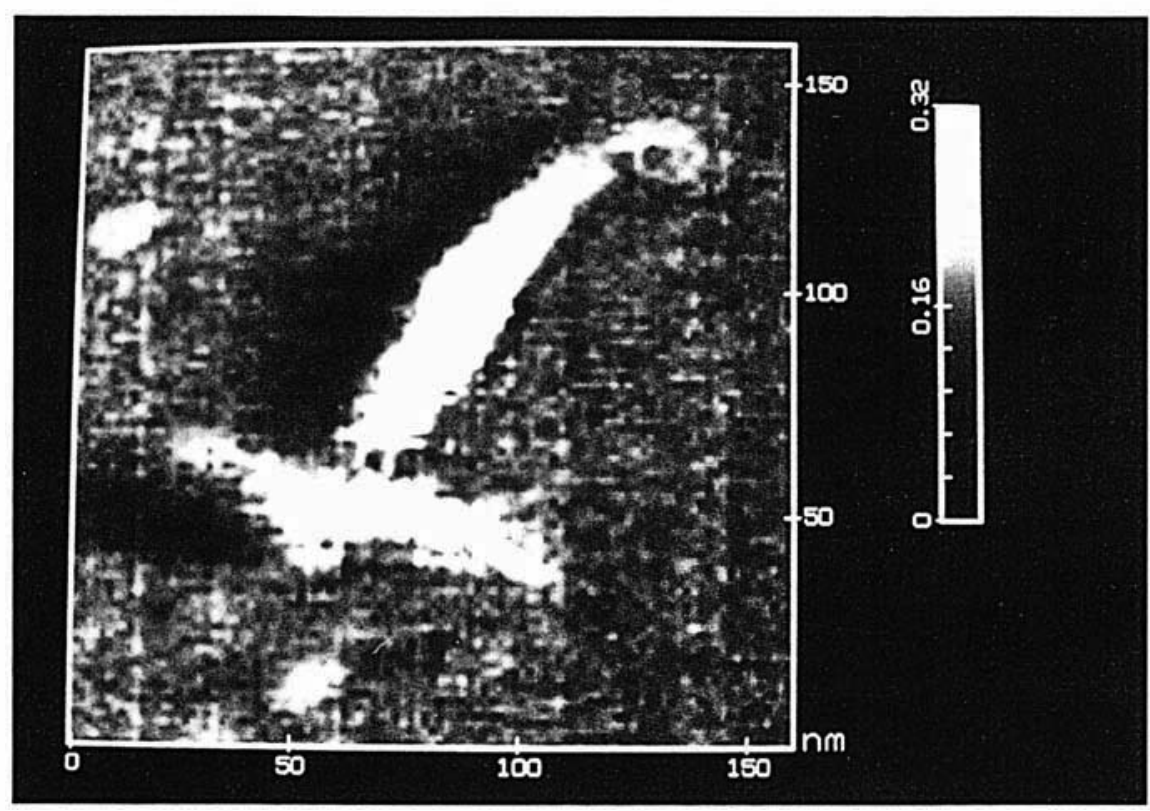

Figure 9. Molécule de laminine visualisée avec une faible pression de levier. Graduations : dimensions en $\mathrm{nm}$.

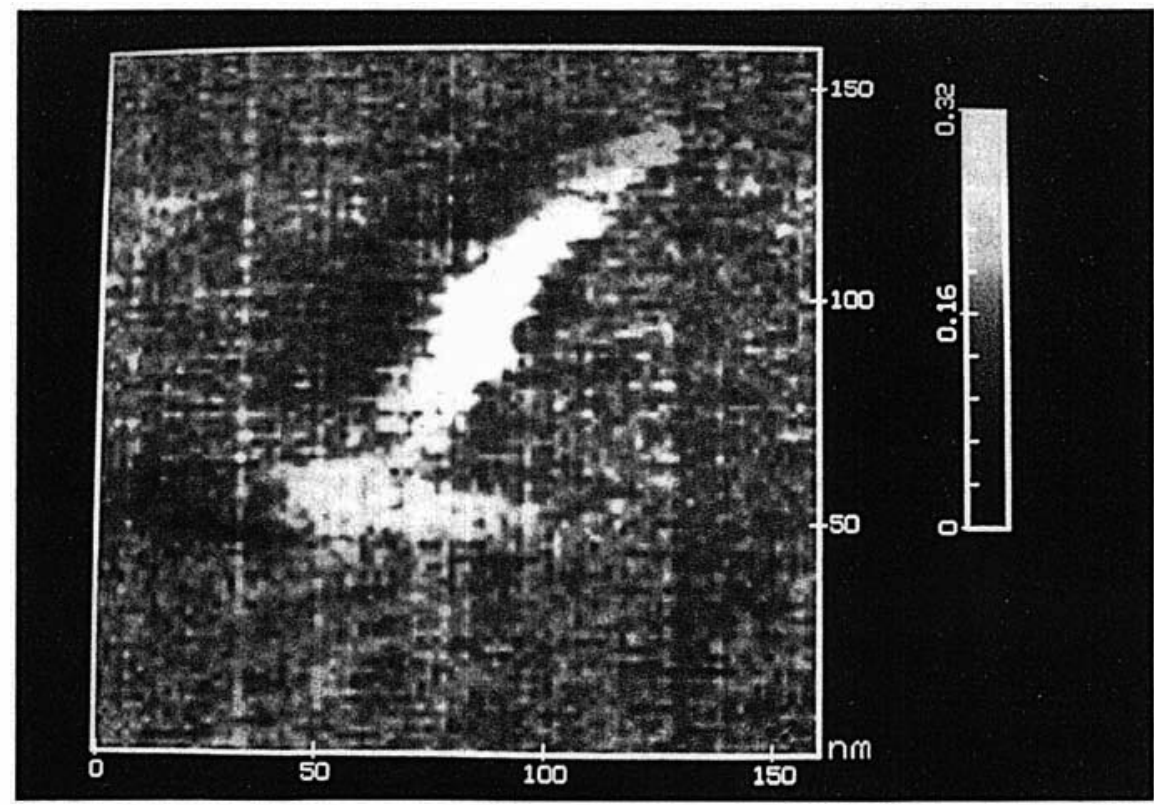

Figure 10. Molécule de laminine visualisée avec une forte pression de 146 échantillon de mica collé avec de la Cyanolith $^{\circledR}$ sur un support en verre et visualisé avec l'AFM. Les deux carrés montrent des zones précédemment visualisées avec une pression de levier plus grande.

On voit nettement que l'aiguille comprime le substrat, rendu mou par la Cyanolith $^{\circledR}$, en lui affectant des déformations qui n'existaient pas auparavant. Il faut noter que le mica est un support très utilisé en microscopic AFM en raison de sa dureté et de sa platitude atomique... Un autre artefact couramment observé est le déplacement du spécimen sous l'aiguille de l'AFM. Une molécule peut être visible lors d'un balayage et invisible lors du suivant. Nous supposons que dans ces cas-là, la molécule a été "écartée " ou " accrochée " par l'aiguille et se déplace avec elle. A plusieurs reprises, nous avons observé des images dont une seule partic était déformée, comme représenté sur la figure 13 (p. 148). Dans ce cas, nous supposons que l'aiguille a rencontré un corps solide qui déforme latéralement le levier et produit ce type d'aberration.

Parfois la topographie de l'échantillon change, suivant que le balayage se fait dans un sens plutôt que dans un autre. Il arrive, surtout à haute résolution, que le balayage "aller " donne une image différente du balayage "retour"; cela peut être expliqué par le fait que le levier est beaucoup plus souple que l'échantillon, comme c'est illustré sur la figure 14 (p. 148).

- Artefacts dus à un traitement numérique du signal

Les images "brutes" obtenues par l'AFM sont souvent parasitées par un bruit de fond électronique ou une vibration de l'aiguille. Pour pouvoir exploiter ces images, il est parfois nécessaire de les filtrer, ce qui introduit de nouveaux artefacts. Les zones noires, situées à la gauche des protubérances visibles sur les figures 8,9 , 12 et qui donnent un aspect ombré à l'image, sont la conséquence d'un tel filtrage.

- Artefacts dus à la géométrie de l'aiguille

La forme de l'aiguille peut avoir un effet dramatique sur les images. Pour 


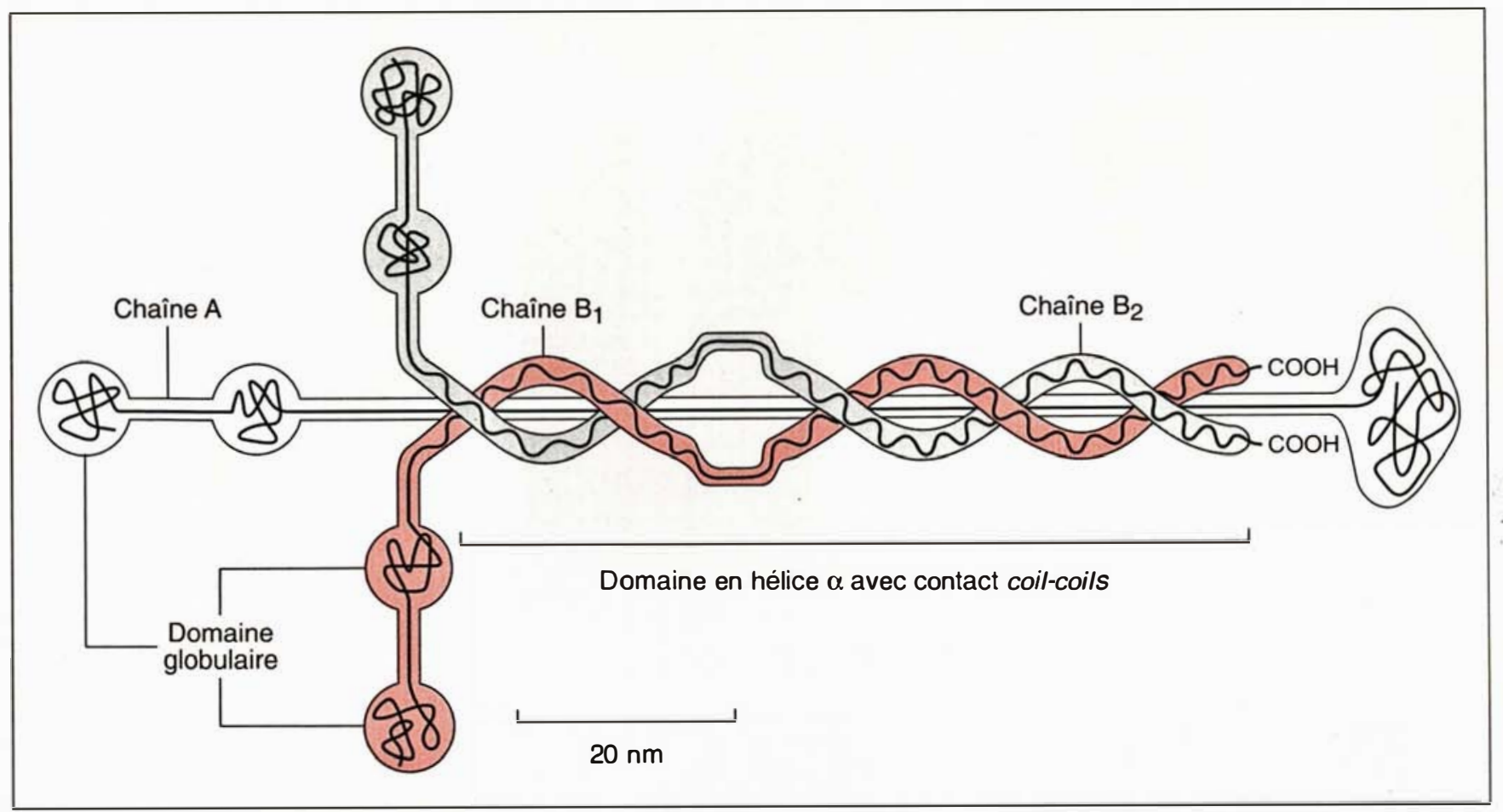

Figure 11. Modèle théorique de la molécule de laminine. (D'après The molecular biology of the cell, p. $820,2^{e}$ éd. 1989).

pouvoir prédire ce type d'artefacts, nous avons simulé un scanning probe microscope sur ordinateur. Il nous est possible de dessiner la pointe ainsi que l'échantillon et d'observer l'image que va fournir le microscope. La figure 15 (p. 148) illustre une simulation faite sur un échantillon en pavés avec une aiguille présentant une double pointe.

\section{Perspectives}

Malgré ses limites et ses artefacts, le microscope à force atomique nous semble être un instrument très prometteur en biologie et, plus particulièrement, en morphologie. Il ne remplacera certainement pas le microscope électronique, mais il permettra l'exploration de structures dans des milieux et des conditions auxquels celui-ci ne peut pas accéder. Les futurs travaux seront certainement axés sur la visualisation de phénomènes dynamiques à l'échelle moléculaire, ainsi que sur l'observation de structures vivantes évoluant dans un milieu aussi proche que possible de leur milieu naturel. Plusieurs groupes travaillent actuellement à la mise au point d'AFM fonctionnant à basse $m / s n^{\circ} 2$, vol. 8, février 92

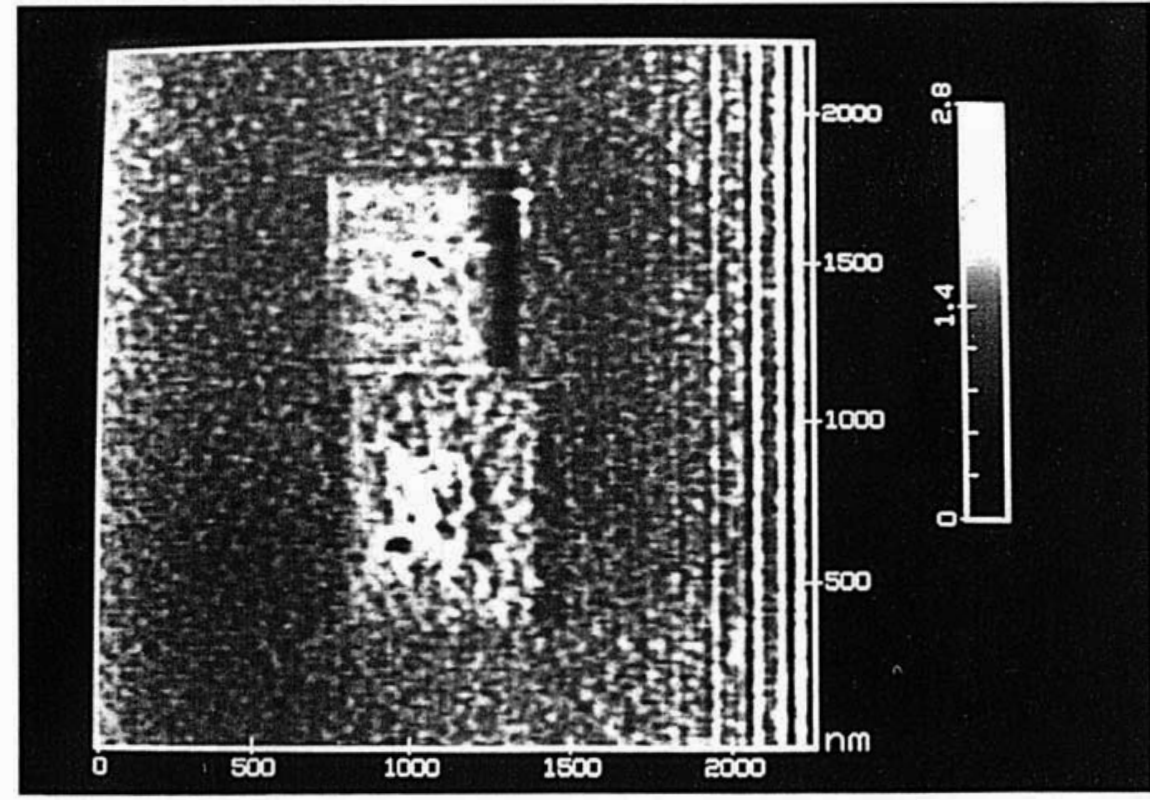

Figure 12. Image montrant les déformations pouvant survenir sur le mica. Les deux carrés visibles sur cette image correspondent à deux zones qui ont été précédemment balayées avec une pression de levier plus élevée. Graduations : dimensions en $\mathrm{nm}$. 


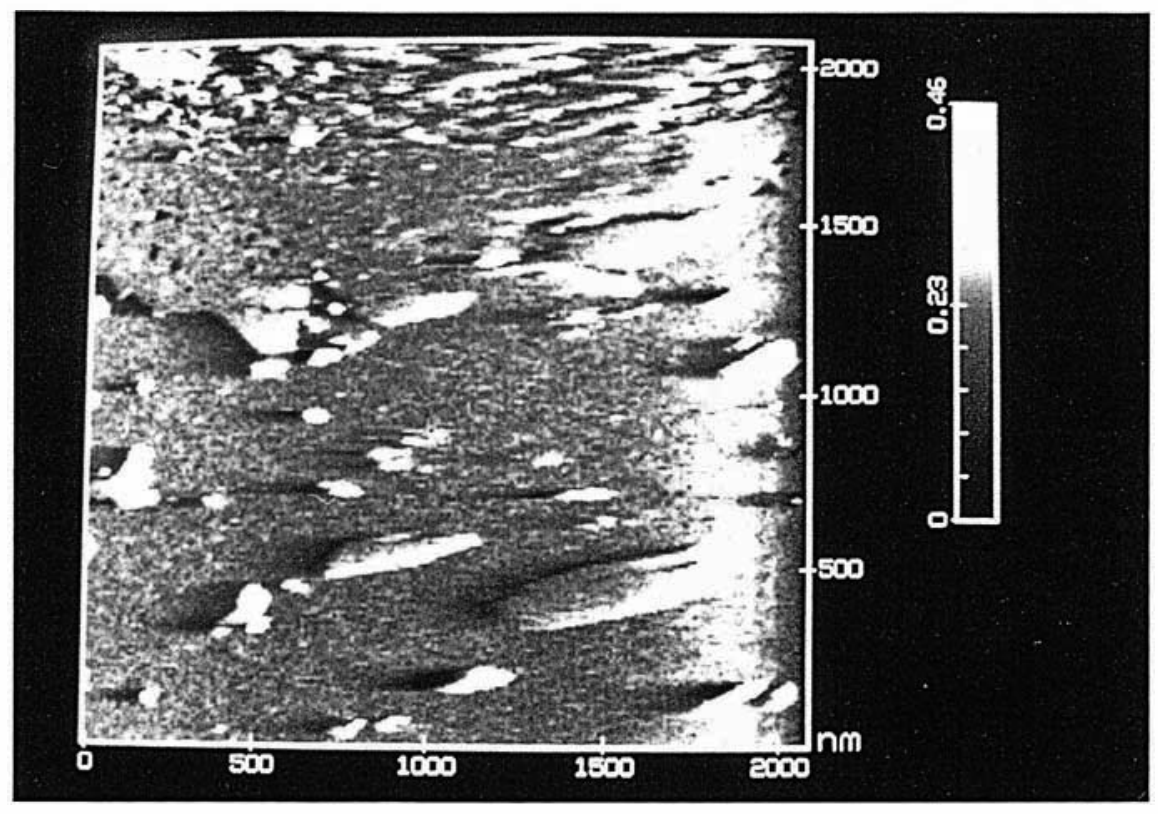

Figure 13. L'AFM n'est pas non plus à l'abri des aberrations. Dans ce cas, une explication possible est que la pointe ait touché un corps solide qui déforme le levier latéralement. Une autre explication serait qu'en certains endroits l'aiguille est plus attirée par l'échantillon qu'en d'autres. Graduations : dimensions en $\mathrm{nm}$.

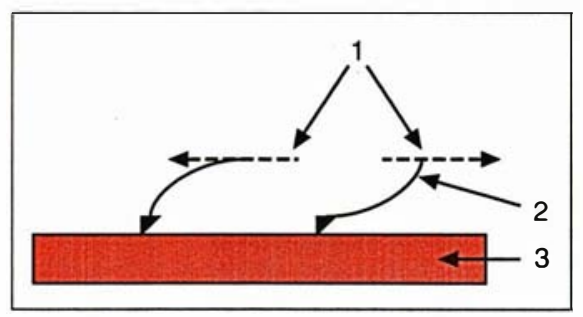

Figure 14. Le levier peut être déformé de manière différente suivant le sens du balayage. 1. directions du balayage; 2 . levier; 3. échantillon.

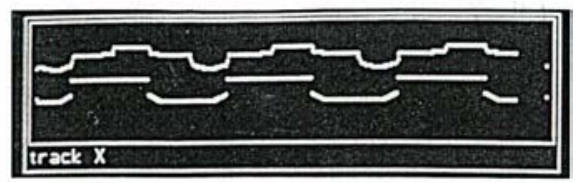

Figure 15. Simulation d'un AFM. La ligne inférieure représente le profil de l'échantillon et la ligne supérieure l'image qu'en donne le microscope lorsque sa pointe se termine par deux température. Ils espèrent ainsi augmenter la résolution du microscope en figeant l'échantillon dans l'azote liquide [14]. Une autre voie de recherche est la construction d'AFM à plusieurs canaux, l'un montrant la topographie et l'autre la température ou la charge électrique de l'échantillon, par exemple.

\section{Conclusion}

Cinq ans après son invention, le microscope à force atomique s'est avéré un puissant outil d'observation des structures biologiques à l'échelle moléculaire. Sa capacité à travailler en milieu liquide laisse entrevoir la possibilité de visualiser des structures vivantes avec une résolution inégalée. Il est aussi le seul instrument existant qui permet l'exploration des propriétés mécaniques d'une seule molécule. Comme tous les microscopes, l'AFM a bien entendu ses défauts et ses arteoublier que ceux-ci dépendent grandement de la méthode de préparation des échantillons et qu'il a fallu trente ans pour perfectionner les techniques de préparation de la microscopie électronique facts, mais nous ne devons pas atomes.

\section{Summary}

Application of the atomic force microscope in biological research

The atomic force microscope (AFM) is a new kind of instrument allowing an atomic resolution on crystals and molecular resolution on some biological materials. Its ability to image samples immersed in a fluid makes it highly interesting for biologists. The working principle of the AFM is based on the scanning of a sample with a tip fixed at the end of a cantilever. A feed-back loop maintains a constant deflection of the cantilever by moving the sample up and down while scanning. All movements of the sample are processed by a computer and the surface of the sample is reconstructed on a screen. We present here images obtained using this microscope on $\mathrm{NaCl}$ crystals, slices of rat striated muscle, erythrocytes immersed in a physiological buffer and molecules of laminin. Like all microscopes, the AFM can interact with the sample and generate modifications of its surface. Several artifacts are presented and explained. Nevertheless, continuous work with this instrument will allow the study of dynamic phenomena of living tissues at high resolutions.

\section{TIRÉS A PART}

S. Kasas 